\title{
Apoyo emocional que brinda el profesional de Enfermería y el afrontamiento de los padres de pacientes pediátricos posoperados con cardiopatía congénita, INCOR 2014
}

\author{
Emotional support provided by nursing professionals and coping of parents of pediatric patients with \\ congenital heart disease after surgery, INCOR 2014.
}

Gladys Norma Manturano Velásquez'1, Keila Ester Miranda Limachi²

\begin{abstract}
RESUMEN
Objetivo: Determinar la relación que existe entre apoyo emocional que brinda el profesional de enfermería y el afrontamiento de los padres de pacientes pediátricos posoperados con cardiopatía congénita. Metodología: El estudio corresponde a un diseño no experimental, tipo cuantitativo, descriptivo, correlacional, de corte transversal. La muestra estuvo compuesta por 44 padres de pacientes pediátricos posoperados con cardiopatía congénita, se utilizó un muestreo no probabilístico. Para la recolección de datos se utilizaron dos instrumentos. Resultados: El $27 \%$ de padres recibe apoyo emocional favorable y el afrontamiento es favorable; el $43 \%$ de padres recibe apoyo emocional desfavorable y el afrontamiento es desfavorable. Aplicando la prueba estadística de chi cuadrado, la relación de las variables fue significativa p: .009; es decir, a mayor apoyo emocional del profesional de enfermería, mejor afrontamiento de los padres de pacientes pediátricos posoperados con cardiopatía congénita. Conclusión: El apoyo emocional, en los momentos de crisis, es de vital importancia para tener un afrontamiento favorable.
\end{abstract}

Palabras clave: Apoyo emocional, afrontamiento, enfermería, cardiopatía congénita.

\begin{abstract}
Objective: To determine the relationship between emotional support provided by the nurse and coping of parents of pediatric patients with congenital heart disease after surgery. Methodology: The study corresponds to a nonexperimental design, quantitative, descriptive, correlational, cross-sectional type. The sample was composed by 44 parents of pediatric patients with congenital heart disease after surgery, a non-probability sampling was used. To collect data two instruments were used. Results: There were: $27 \%$ of parents receive positive emotional support and coping is favorable; $43 \%$ of parents receive adverse emotional support and coping is unfavorable. Applying statistical chi-square test, the relationship between the variables was significant p: .009; ie, the greater the emotional support of the nurse better coping parents of pediatric patients with congenital heart disease after surgery. In conclusion, emotional support in times of crisis it is vital to have a positive coping.
\end{abstract}

Keywords: Emotional support; coping, nursing, congenital heart disease. 


\section{INTRODUCCIÓN}

El presente siglo ha sido testigo de numerosos avances de la tecnología médica que han permitido el control parcial o total de diferentes enfermedades que acortaban la esperanza de vida de las personas, permitiendo así que ahora se viva más y en mejores condiciones de salud (Matón, 1993).

En la actualidad, a nivel mundial, Latinoamérica y nacional se estima que la incidencia global de las cardiopatías congénitas varía desde 8 hasta 10 cardiopatías al año por cada 1.000 recién nacidos; gracias a los avances en cardiología y cirugía cardiaca, el $80-85 \%$ de los niños afectados de cardiopatía llegarán a la vida adulta. Algunos totalmente curados, otros presentarán secuelas sin mayor importancia; un tercer grupo necesitará vigilancia estrecha y nuevas intervenciones (Zaldívar, 2003).

El manejo es altamente complejo, los pacientes pediátricos con cardiopatías congénitas ingresan a la Unidad de Cuidados Intensivos por el alto riesgo de complicaciones fisiopatológicas; la tensión psíquica que tienen que enfrentar el paciente y su familia también es muy compleja. Cuando se comunica a los padres que su hijo tiene una cardiopatía, ellos sienten un gran impacto emocional de pánico, preocupación, ansiedad y miedo, también surge una necesidad imperiosa de comprender y saber más sobre la enfermedad y sobre cómo es vivir con un niño con cardiopatía congénita, además una gran preocupación por la vida y el porvenir de su hijo (Rodríguez, 2007). Es entonces cuando la familia, núcleo integrador, se manifiesta en una red de apoyo eficaz para el individuo en el ajuste a la vida social y, en especial, en la recuperación de las secuelas de la enfermedad, convirtiéndose en el sostén imprescindible en situaciones de crisis (Alli, 2000).

El enfermero se convierte, en este ambiente, $z$ en un profesional clave en el apoyo emocional al paciente y familia, creando un espacio de escucha para proporcionar seguridad, aceptación y ánimo, antes y durante los momentos de tensión, mediante un acercamiento mediante el diálogo sincero y abierto, que favorecerá la expresión de los sentimientos y pensamientos para permitir el afrontamiento a esta nueva situación, ya que el afrontamiento es resultado de la interacción entre el sujeto y su contexto, según el tipo de problema a resolver (Barrera, 2008).

El acto de cuidar aplicado por el enfermero no se limita solo a satisfacer las necesidades físicas más inmediatas; pues como refiere Colliere (1993), "cuidar es ante todo un acto de vida, representa una infinita variedad de actividades dirigidas a mantener y conservar la vida y a facilitar las condiciones para la realización del proceso vital humano". Asimismo, Domínguez (1986) argumentó: "cuidar significa movilizar las capacidades del ser humano bien sea en estado de salud o enfermedad, teniendo en cuenta sus costumbres para lograr su crecimiento y desarrollo desde el nacimiento hasta la muerte", cuidar implica la realización de actividades en diferentes niveles de complejidad, usando la tecnología según la gravedad de la enfermedad de pacientes sometidos a cirugía cardíaca, quienes requieren el ingreso a unidades especiales para el manejo del posoperatorio inmediato, mediante la colocación de múltiples sondas y catéteres implantados provisionalmente, realizando así una estrecha vigilancia sobre toda la economía, especialmente sobre la función cardíaca, cuya vigilancia la realizan los médicos intensivistas (Villagra, 2010). Toda esta situación genera un contexto de sufrimiento y dolor en los padres, con la necesidad de recibir afecto, palabras de aliento e información sobre lo que está aconteciendo. Por eso, se planteó el presente estudio, para indagar sobre la relación que existe entre el apoyo emocional del profesional de enfermería y el afrontamiento de los padres de pacientes pediátricos posoperados con cardiopatía congénita, INCOR, 2013.

Según Galindo (2006), la enfermera, en su condición de cuidadora al pie de la cama, se convierte en estas circunstancias en un profesional clave para la atención a los niños y a sus padres, proporcionando apoyo emocional y brindando soporte a los padres en los momentos más críticos; en cuyas circunstancias se observa un desgaste emocional de los padres por no estar preparado para afrontar esta realidad; toda enfermedad es incomprensible, pero es especialmente injustificable cuando ataca a un niño.

En respuesta a esta situación descrita, el presente estudio aparece con el objetivo de determinar la relación que existe entre el apoyo emocional del profesional de enfermería y el afrontamiento de los padres de pacientes pediátricos posoperados con cardiopatía congénita, INCOR 2013.

Cuando la enfermedad cardíaca aparece en el lactante, niño o adolescente, no solo afecta a la persona que la sufre, sino a todo su entorno: padres, hermanos, amigos, etc. No es un trabajo imposible, sino un camino por recorrer, paso a paso; la resistencia personal puede verse afectada, también beneficiada con el apoyo emocional al enfermo y a la familia. Solo si se cuenta con una información clara y detallada del equipo médico y de enfermería se puede tomar conciencia sobre el problema para enfrentarse a él de una forma emocionalmente eficaz. 
Apoyo emocional que brinda el profesional de Enfermería y el afrontamiento de los padres de pacientes pediátricos posoperados con cardiopatía congénita, INCOR 2014

\section{MATERIAL Y MÉTODOS}

El presente trabajo de investigación fue cuantitativo, descriptivo, correlacional de corte transversal y de diseño no experimental. La población estuvo constituida por 113 padres de los pacientes pediátricos posoperados con cardiopatía congénita. Se seleccionó una muestra de 44 padres a través de un muestreo no probabilístico considerando los criterios de inclusión y exclusión. Para medir el apoyo emocional, la investigadora elaboró el cuestionario basado en los diagnósticos del NANDA 20122014. Para medir el afrontamiento de los padres, se adaptó 2 instrumentos: escala de afrontamiento para adolescentes elaborados por Fydenberg y Compas en el año 1997, y el inventario de estrategias de afrontamiento para adolescentes y adultos elaborados por Moos, en el año 1993. Ambos cuestionarios fueron validados por profesionales con experiencia en investigación y con manejo de pacientes pediátricos con cardiopediatría. Para medir la confiabilidad de los mismos, se realizó una prueba piloto a 15 padres con características similares a la muestra. Se aplicó la prueba de Alpha de Cronbach, obteniéndose los puntajes de 0.953 y 0.831 para los instrumentos de apoyo emocional y afrontamiento, respectivamente.

\section{RESULTADOS}

Tabla 1

Datos sociodemográficos de los padres de pacientes pediátricos posoperados con cardiopatía congénita, INCOR 2013.

\begin{tabular}{|c|c|c|c|}
\hline \multirow[t]{3}{*}{ Edad } & 23 - 31 años & 8 & 18.2 \\
\hline & 32 - 40 años & 27 & 61.4 \\
\hline & $41-$ mas & 9 & 20.5 \\
\hline \multirow[t]{3}{*}{ Hijos } & $1-2$ hijos & 28 & 63.6 \\
\hline & $3-4$ hijos & 15 & 34.1 \\
\hline & $5-$ mas & 1 & 2.3 \\
\hline \multirow[t]{3}{*}{ Procedencia } & Costa & 33 & 75.0 \\
\hline & Sierra & 10 & 22.7 \\
\hline & Selva & 1 & 2.3 \\
\hline \multirow[t]{6}{*}{ Nivel de instrucción } & Primaria Comp. & 5 & 11.4 \\
\hline & Secund. Comp. & 6 & 13.6 \\
\hline & Secund.incompleta & 4 & 9.1 \\
\hline & Sup. Tec. Compl. & 12 & 27.3 \\
\hline & Sup. Tec. Incomp. & 5 & 11.4 \\
\hline & Sup. Uni. Comp. & 12 & 27.3 \\
\hline \multirow[t]{3}{*}{ Religión } & Católico & 37 & 84.1 \\
\hline & Cristiano & 6 & 13.6 \\
\hline & Adventista & 1 & 2.3 \\
\hline \multirow[t]{4}{*}{ Paciente } & Recién nacido & 2 & 4.5 \\
\hline & Lactante & 26 & 59.1 \\
\hline & Preescolar & 8 & 18.2 \\
\hline & Escolar & 8 & 18.2 \\
\hline \multirow[t]{3}{*}{ Edad } & 23 - 31 años & 8 & 18.2 \\
\hline & 32 - 40 años & 27 & 61.4 \\
\hline & 41 - más & 9 & 20.5 \\
\hline
\end{tabular}




\begin{tabular}{llll}
\hline Hijos & $1-2$ hijos & 28 & 63.6 \\
& $3-4$ hijos & 15 & 34.1 \\
& $5-$ más & 1 & 2.3 \\
\hline Procedencia & Costa & 33 & 75.0 \\
& Sierra & 10 & 22.7 \\
& Selva & 1 & 2.3 \\
\hline Nivel de instrucción & Primaria Comp. & 5 & 11.4 \\
& Secund. Comp. & 6 & 13.6 \\
& Secund.incompleta & 4 & 9.1 \\
& Sup. Tec. Compl. & 12 & 27.3 \\
& Sup. Tec. Incomp. & 5 & 11.4 \\
& Sup. Uni. Comp. & 12 & 27.3 \\
\hline Religión & Católico & 37 & 84.1 \\
& Cristiano & 6 & 13.6 \\
& Adventista & 1 & 2.3 \\
\hline Paciente & Recién nacido & 2 & 4.5 \\
& Lactante & 26 & 59.1 \\
& Preescolar & 8 & 18.2 \\
& Escolar & 8 & 18.2 \\
\hline
\end{tabular}

En la tabla 1 se observa que el $81.9 \%$ de los participantes, en la investigación, tiene de 32 años a más, el $81.8 \%$ está representado por las madres de los pacientes pediátricos, el $63.6 \%$ tiene entre uno y dos hijos, el $75 \%$ proviene de la costa, el $54.6 \%$ tiene estudios a nivel superior y el $84.1 \%$ profesa la religión católica.

Tabla 2

Tipos de apoyo emocional de los padres de pacientes pediátricos posoperados con cardiopatía congénita del INCOR, 2013.

\begin{tabular}{llll}
\hline Tipos apoyo emocional & & $\mathrm{N} .{ }^{\circ}$ & $\%$ \\
\hline \multirow{2}{*}{ AE Afectivo } & Desfavorable & 21 & $47.7 \%$ \\
& Favorable & 23 & $52.3 \%$ \\
AE Tangible & Desfavorable & 23 & $52.3 \%$ \\
& Favorable & 21 & $47.7 \%$ \\
AE Informativo & Desfavorable & 27 & $61.4 \%$ \\
& Favorable & 17 & $38.6 \%$ \\
\hline
\end{tabular}

La tabla 2 muestra que, en cuanto al apoyo emocional que recibieron los padres, el apoyo afectivo alcanzó un $52.3 \%$ en la categoría favorable y el apoyo tangible e informativo presentó $52.3 \%$ y $61.4 \%$ respectivamente, en la categoría de desfavorable. 
Apoyo emocional que brinda el profesional de Enfermería y el afrontamiento de los padres de pacientes pediátricos posoperados con cardiopatía congénita, INCOR 2014

Tabla 3

Tipos de afrontamiento de los padres de pacientes pediátricos posoperados con cardiopatía congénita del INCOR, 2013

\begin{tabular}{llll}
\hline Tipos de afrontamiento & & $\mathrm{N} .{ }^{\circ}$ & $\%$ \\
\hline \multirow{2}{*}{ AF Problema } & Desfavorable & 23 & $52.3 \%$ \\
& Favorable & 21 & $47.7 \%$ \\
AF Emoción & Desfavorable & 26 & $59.1 \%$ \\
& Favorable & 18 & $40.9 \%$ \\
AF Evitativo & Desfavorable & 28 & $63.6 \%$ \\
& Favorable & 16 & $36.4 \%$ \\
\hline
\end{tabular}

La tabla 3 muestra que, en cuanto al afrontamiento de los padres, los mayores porcentajes se ubicaron en la categoría desfavorable, con un $52.3 \%$ para el afrontamiento dirigido al problema, un $59.1 \%$ para el afrontamiento dirigido a la emoción y un $63.6 \%$ para el afrontamiento evitativo. Sin embargo, es importante resaltar que en la categoría favorable, el mayor porcentaje corresponde al afrontamiento dirigido al problema, con un $47.7 \%$.

Tabla 4

Apoyo emocional que brinda el profesional de enfermería y su relación con el afrontamiento de los padres de pacientes pediátricos posoperados con cardiopatía congénita, INCOR 2013.

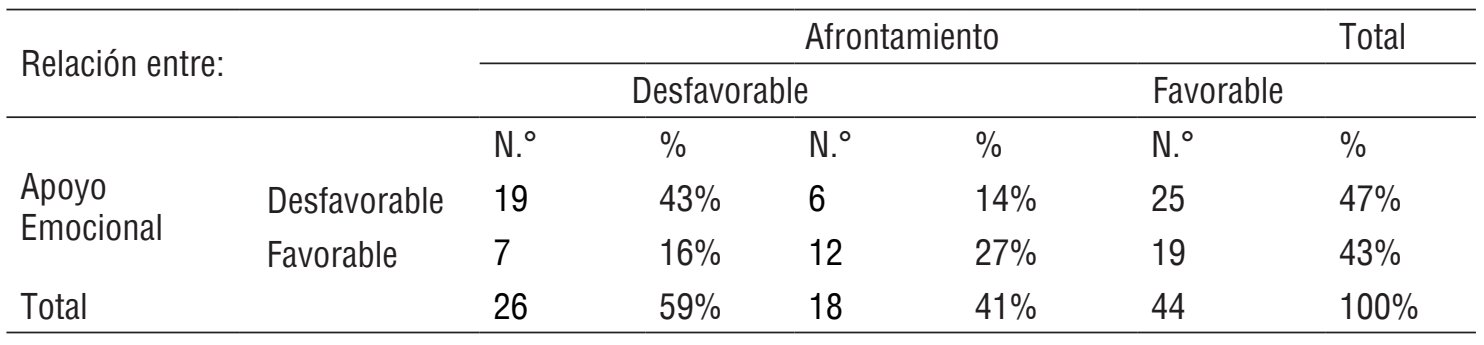

Nota: $\mathrm{X}^{2}=6.848^{\mathrm{a}} ; \mathrm{Gl}=1 ; \mathrm{sig}=0.009$

La tabla 4 presenta la prueba de Chi cuadrado, para las dimensiones del apoyo emocional y el afrontamiento de los padres evaluados a un nivel de significancia de 0.05 , evidenciándose que la dimensión de apoyo afectivo tiene una relación significativa

con $\mathrm{X}^{2}=6.848, \mathrm{p}=0.005$. Las dimensiones de apoyo tangible e informativo, no presentaron relación significativa, por tener valores superiores a 0.05 .

\section{Tabla 5}

Relación entre las dimensiones de apoyo emocional que brinda el profesional de enfermería y afrontamiento de los padres de pacientes pediátricos posoperados con cardiopatía congénita, INCOR - 2013.

\begin{tabular}{lll}
\hline Dimensiones & $\mathrm{X}^{2}$ & $\mathrm{p}$ \\
\hline Apoyo emocional afectivo & 7.943 & .005 \\
Apoyo emocional tangible & 2.187 & .139 \\
Apoyo emocional informativo & 3.678 & .055 \\
\hline
\end{tabular}

En la tabla $\mathrm{N}^{\circ} 5$ se puede observar que luego de realizar un análisis de las dimensiones de apoyo emocional y el afrontamiento de los padres, se encontró relación significativa entre la dimensión apoyo emocional afectivo y el afrontamiento de los padres; asimismo, las dimensiones apoyo emocional tangible e informativo presentan valores de $p>0.05$, la cual indica que no existe relación significativa con el afrontamiento de los padres de los pacientes pediátricos. 


\section{DISCUSIÓN}

La esfera emocional es elemental en el desarrollo humano y, más aún, cuando se experimenta una situación de crisis y/o sufrimiento, al saber que un hijo padece una cardiopatía congénita y va a ser intervenido quirúrgicamente; desde el momento de la internación en un ambiente hospitalario, se experimenta un gran impacto emocional influyendo en la dinámica psíquica; los familiares tienen que lidiar con personas desconocidas, con culturas y normas diferentes, dejan de ser sujetos para pasar a ser objetos del conocimiento $\mathrm{y}$ al poder del médico (Silva, 2005). En lo que respecta al ofrecimiento de apoyo emocional, los profesionales de enfermería son considerados los más aptos para otorgarlo dentro de todo el equipo de salud (Gagnon, Waghorn y Covell, 1997).

Asimismo, según Krausf (1992), las enfermeras tienen mayor contacto con los pacientes y con el acompañante; por lo tanto, son un elemento clave de los cuidados referente al apoyo emocional. Sin embargo, los resultados del estudio muestran que el $56.8 \%$ de los familiares recibe un apoyo emocional desfavorable y el $43.2 \%$, apoyo emocional favorable. Este resultado podría deberse a diversos factores, por ejemplo, horarios de visita restringidos que limita el contacto continuo con los familiares, grado de responsabilidad con el monitoreo minucioso de la vida del paciente, donde se da prioridad a los aspectos científicos/tecnológicos y a la alta demanda de información a los familiares por tener un paciente crítico, entre otros aspectos

Los resultados del estudio son similares al trabajo realizado por Franco, R. (2004), titulado Percepción del familiar del paciente crítico respecto a la intervención de enfermería durante la crisis situacional en la Unidad de Cuidados Intensivos en el Hospital Nacional Edgardo Rebagliati, donde muestra que un $37.5 \%$ de los familiares refirió una percepción favorable respecto a que las enfermeras utilizan un lenguaje claro y sencillo al responder alguna inquietud, explican los procedimientos, prestan atención, se muestran atentas y comprensivas, el $33.3 \%$ desfavorable y $29.2 \%$ indiferente; respecto a: las enfermeras no orientan a la familia sobre la forma de proceder frente a los sentimientos de angustia, no tienen un tono cálido al hablar con los familiares y no tranquilizan con palabras de aliento.

En este contexto, se puede indicar que la hospitalización de un niño, con cardiopatía congénita en la UCI, constituye un factor que altera la homeostasia psicoemocional de la familia, la cual está relacionada con la percepción que estos tengan sobre dicha situación que, a su vez, es influida por la información, el grado de instrucción, el sexo, personalidad, la comprensión de la situación, la intolerancia, experiencias anteriores, las necesidades, etc.

Por otro lado, este estudio indica que de los tres tipos de apoyo emocional, según la categoría de favorable, el de mayor porcentaje es el apoyo afectivo: el 52.3\%, seguido por el apoyo tangible: el $47.7 \%$ y, por último, el apoyo informativo: el $38.6 \%$ (ver tabla 2). En el estudio de investigación realizado por Hannah (2005), titulado Ayuda de hermanos de niños con retraso mental, los resultados son similares a nuestro estudio, de 100 niños, dividido: 50 con hermano con retraso mental y 50 sin hermano con retraso mental, el mayor porcentaje de la muestra recibieron apoyo afectivo de sus hermanos, mientras el apoyo informativo y ayuda tangible fueron poco significativos; por todo ello, se puede deducir que el ser humano, desde que nace, es un ente afectivo; por tanto, su necesidad de dar y recibir afecto es relevante.

Al respecto, en el estudio de Llopis (2011): Patrones de cambio en las familias con niños con cardiopatía congénita, el $32.4 \%$ de los familiares indicó que su mayor necesidad es el apoyo informativo del profesional de enfermería; concluyó que la información es necesaria durante todo el proceso de la enfermedad. Ante estas diferencias, podemos afirmar que los padres son seres con diferentes necesidades, actitudes, patrones culturales, etc.

Por otro lado, ante la situación de sufrimiento o padecimiento, los padres ponen en marcha los mecanismos de actuación para paliar los daños, definidos como intentos de afrontamiento, se entienden como un conjunto de esfuerzos cognitivos y comportamentales, continuamente cambiantes que son utilizados para afrontar demandas valoradas por el individuo como excesivas o desbordantes respecto a los recursos de que dispone o cree disponer (Lazarus y Folkman, 1986). Al respecto se proponen dos estrategias generales para afrontar el estrés: 1) Afrontamiento directo o acción directa, una estrategia aplicada externamente a la fuente ambiental de estrés para intentar dominar la transacción estresante con el ambiente; y 2) Afrontamiento indirecto o acción paliativa, una estrategia aplicada a las propias emociones, en la que la persona intenta reducir el trastorno cuando es incapaz de controlar el ambiente o cuando la acción es demasiado costosa (Langer y Campero,1998).

En esta investigación, se considera tres tipos de estrategias para afrontar una situación de estrés: 1) 
Afrontamiento dirigido al problema o llamado activo conductual, 2) Afrontamiento dirigido a la emoción o activo cognitivo y 3) Evitativo o improductivo (Fridemberg, 1997). En este estudio se muestra que del total de padres de paciente pediátricos posoperados con cardiopatía congénita, un $59.1 \%$ presenta un afrontamiento desfavorable y el $40.9 \%$, afrontamiento favorable. Estos resultados guardan relación directamente proporcional: a mayor apoyo emocional, los padres tendrán un afrontamiento más favorable, si disgregamos los tipos de afrontamiento, según la categoría de favorable, se observa que el afrontamiento dirigido al problema es el de mayor relevancia: el $47.7 \%$, seguido por afrontamiento dirigido a la emoción con $40.9 \% \mathrm{y}$, por último, afrontamiento evitativo: $36.4 \%$ (ver tabla 3 ).

Según Leiter (2001) y Santos (2002), las personas que se esfuerzan por cambiar la situación estresante o afrontamiento dirigido al problema, presentan más satisfacción y menos sensación de cansancio emocional; asimismo, indican que las personas quienes centran su afrontamiento a la emoción, presentan cansancio emocional y menor autoeficacia, asimismo, la utilización de la estrategia evitación correlaciona los tres factores característicos: cansancio emocional, despersonalización y falta de realización personal.

Al respecto, Llopis (2008) realizó un estudio titulado Redes sociales de padres de niños afectados con cardiopatía congénita, los padres para lograr el afrontamiento recorren un largo camino el cual se inicia en el momento que reciben la noticia de la enfermedad de su hijo, las ilusiones y expectativas se ven alteradas en el momento del nacimiento, mientras que, para la aceptación, puede pasar meses e incluso años en este proceso conocido como duelo.

Kern (2007) refiere que la familia busca adaptarse a la enfermedad en sus diferentes fases (diagnóstico, tratamiento), produciendo diversas reacciones, de acuerdo con su grado de vulnerabilidad o su grado de resiliencia, estos dependen de numerosos factores: experiencia en situaciones de crisis y problemas médicos, estatus socioeconómico, nivel de conocimientos, calidad de los servicios sanitarios y educativos, y sistemas de apoyo.

Por todo eso, es de vital importancia reconocer la labor que desempeña el profesional de enfermería en cuanto al apoyo emocional, por ser un elemento clave en esta esfera en momentos críticos y/o de sufrimiento.
En cuanto a la relación que existe entre apoyo emocional que brinda el profesional de enfermería con el afrontamiento de los padres de pacientes pediátricos posoperados con cardiopatía congénita, se observa de 44 padres (el 100\%), el 27\% de padres presenta apoyo emocional y afrontamiento favorable, mientras el $43 \%$ presenta apoyo emocional y afrontamiento desfavorable (ver tabla 4). Aplicando la prueba estadística de chi cuadrado, la relación de las variables es significativa p: .009; es decir, el mayor apoyo emocional que brinda el profesional de enfermería mejora el afrontamiento de los padres de pacientes pediátricos posoperados con cardiopatía congénita.

Al respecto, Pelaez (2007) indica que el apoyo emocional es un conjunto de técnicas, para crear un espacio de escucha y así proporcionar seguridad, aceptación y ánimo en momentos de tensión, sobre todo cuando un padre recibe la noticia que su hijo tiene una cardiopatía congénita y urge ser hospitalizado en la UCI; ellos suelen sentir temor y angustia al ver equipos altamente tecnificados que rodean a su hijo y, sobre todo, ante un pronóstico incierto de su estado de salud, de ahí que se reconoce la necesidad de apoyar emocionalmente a los padres en el manejo de la crisis que experimentan.

La enfermera, parte del equipo de salud que conoce al paciente, se encuentra en circunstancias claves para proporcionar atención integral al paciente $\mathrm{y}$ a sus padres para ayudar a funcionar las estrategias de afrontamiento y hacer frente al estrés y al dolor psíquico (Carmen, 2010).

Por otro lado, en cuanto a la primera dimensión del apoyo emocional, el $32 \%$ presenta un apoyo emocional afectivo favorable y un afrontamiento favorable, mientras el $39 \%$ de padres presenta un apoyo emocional afectivo desfavorable y afrontamiento desfavorable.

Aplicando la prueba estadística de chi cuadrado, la relación de las variables es significativo p: .005; es decir, el mayor apoyo emocional afectivo que brinda el profesional de enfermería mejora el afrontamiento de los padres de pacientes pediátricos posoperados con cardiopatía congénita.

Esto evidencia que la demanda del apoyo afectivo es alto, porque somos seres afectivos en todos la etapas de nuestra vida, con la necesidad de ser amados y cuidados (Llopis, 2008). 
Asimismo las enfermeras pueden ayudar a disminuir, en gran magnitud, la tensión y sufrimiento a través del apoyo afectivo, haciendo que funcione como amortiguador en este momento crítico, considerando que la enfermería es la profesión que tiene más oportunidad de interactuar constantemente con la familia de estos niños, permitiendo conocer las características propias del proceso de afrontamiento (Monroy, 2010).

En el presente estudio, en la dimensión afectiva, se considera los siguientes indicadores: tono de voz, lenguaje claro, facilitar a que se sientan a gusto, ayudar a dominar sus emociones, recibir expresiones de elogio/respeto, comentar su situación afectiva actual, compartir otras experiencias, escuchar sus sentimientos y creencias, ayudar a identificar su reacción habitual, reconociendo sus sentimientos de ansiedad y tristeza, le ayudan en la toma de decisiones; esto nos permite promover un proceso de adaptación afectiva ante esta nueva situación. El estudio revela que, ante las preguntas planteadas en la encuesta, según el tipo escala de Likert las de mayor puntaje son: ¿el lenguaje que utiliza la enfermera es apropiado? El $59 \%$ de padres respondió siempre; a la pregunta ¿las enfermeras brindan facilidades para que se sientan a gusto en el ambiente hospitalario?, el 52\% de padres respondió siempre y a la pregunta planteada ¿el tono de voz de las enfermeras es agradable?, el 50\% de padres respondió casi siempre; entre las preguntas de menor porcentaje, figura la pregunta ilas enfermeras comentan con usted otras experiencias?, ante la cual solo el $18 \%$ de padres indica siempre; seguido por la pregunta, ¿las enfermeras comentan con usted la situación afectiva por lo que está atravesando?, el 20\% de padres respondió siempre; estas cifras sustentan que los profesionales de enfermería en INCOR son entes que brindan apoyo afectivo en todas las etapas del cuidado del paciente.

Además, cuando los padres perciben la preocupación del profesional de enfermería por la salud de su hijo, se sienten más tranquilos y reconfortados.

Asimismo, la segunda dimensión: apoyo emocional tangible, los resultados indican que el $25 \%$ recibe un apoyo emocional tangible y un afrontamiento favorable, mientras que la cifra más alta es $36 \%$ de los padres, quienes no reciben un apoyo emocional tangible y el afrontamiento es desfavorable, según la prueba estadística de chi cuadrado la significancia asintótica es p: 0.139 , el cual es mayor a nivel de significancia 0.05 ; por lo tanto, se rechaza la hipótesis alterna y se puede concluir que no existe relación significativa entre apoyo emocional tangible con el afrontamiento de los padres de paciente pediátricos posoperados con cardiopatía congénita.

Considerando que el apoyo tangible se refiere en dar servicios como la atención domiciliaria, cuidar a alguien, así como también a la disponibilidad de recursos materiales y operacionales (Valdez, 1990). Asimismo, es fundamental remarcar que, en el estudio, los indicadores de apoyo emocional tangible son: recibe ayuda cuando usted lo requiere, existe disponibilidad para que lo ayuden, la expresión corporal es adecuada, permanecen con usted en los períodos de crisis, favorecen la conversación o el llanto para disminuir la respuesta emocional, son tolerantes cuando usted está alterado, cuando requieren comunicarse con usted y observan que usted está agotado saben esperar.

Ante las preguntas planteadas en el estudio, para esta dimensión, la de mayor relevancia es la pregunta: ¿cuándo está en contacto con las enfermeras sus gestos y actitudes son cordiales? De los cuales, el $48 \%$ de padres respondió siempre, seguido por la pregunta, ¿las enfermeras permanecen con usted y le proporcionan palabras de aliento en los momentos de difíciles? El $43 \%$ de padres indica siempre, mientras que ante las pregunta con menor frecuencia son: ¿las enfermeras le ayudan cuando usted lo necesita dándole alternativas o sugerencias? y ante la pregunta ¿las enfermeras favorecen la conversación o el llanto para disminuir su reacción de desesperación/ crisis? Solo $25 \%$ de padres respondió siempre. Cabe resaltar la predisposición permanente del profesional de enfermería para brindar este apoyo, muchas veces no es percibida por los padres, por el alto nivel de ansiedad y estrés enfrentado; asimismo, la prioridad que otorga la enfermera al paciente, por ser de manejo tan complejo y riguroso en todos los procesos de atención directa, coloca en desventaja a este tipo de apoyo.

En la tercera dimensión, apoyo emocional informativo y el afrontamiento, el $23 \%$ dice que el apoyo emocional informativo y el afrontamiento es favorable, el $43 \%$ afirma que el apoyo emocional informativo y el afrontamiento es desfavorable; según la prueba estadística chi cuadrado, la sig, asintótica bilateral es 0.055 , la cual es ligeramente mayor a nivel de significancia 0.05 ; por lo tanto, se rechaza la hipótesis alterna y se concluye que no existe relación significativa entre apoyo emocional informativo con el afrontamiento de los padres de pacientes pediátricos posoperados con cardiopatía congénita, estos resultados reflejan la necesidad marcada de información durante todos los procesos de la enfermedad que viene a hacer una forma 
habitual de respuesta ante situaciones de estrés, por eso el profesional de enfermería debe estar alerta ante esta necesidad de búsqueda de información repetida, muchas veces asociada a rasgos de vigilancia rígida ante la alta reactividad frente al estrés y la ansiedad de los padres; para valorar el apoyo informativo se consideró 5 preguntas: ¿cuándo usted siente que necesita de consejos por parte de la enfermera, ella se la brinda?, ¿recibe información de las enfermeras cuando usted lo solicita?, ¿cuándo se siente estresado y no puede manejar la situación, recibe información de las enfermeras para superarla?, ¿las enfermeras le ayudan a coordinar con otros profesionales?, ¿las enfermeras le proporcionan apoyo durante la negación, ira y desesperación? De los preguntas consideradas en el estudio, las de mayor ocurrencia fueron: ¿Recibe información de las enfermeras cuando usted lo solicita?, el 52\% de padres respondió siempre, seguida por ¿cuándo usted siente que necesita de consejos por parte de la enfermera, ella se los brinda?, el $36 \%$ de padres respondió siempre, y la de menor frecuencia: ¿las enfermeras le proporcionan apoyo durante la negación, ira y desesperación? Solo $17 \%$ de padres respondió que siempre.

Uno de los factores que incrementan el resultado desfavorable es que los padres, en esta situación, son más exigentes en la información, por eso algunas veces refieren no haber entendido lo supuestamente ya explicado; a su vez, también requieren interpretaciones y reinterpretaciones, considerando que la ansiedad y la negación no les permite recordar la referencia brindada. A su vez, remarcamos que la información es una fuente importante de apoyo, pues permite que los miembros de la familia tomen decisiones conscientes, cabe mencionar que entre el emisor y el receptor del mensaje deben poseer un canal de comunicación abierto (Simponiato, 2008).

Al respecto, Mosquera (2012), en el estudio realizado Estilos de afrontamiento en madres de hijo e hijas con alteraciones congénitas, se concluye que el nacimiento de un hijo o hija crea expectativas positivas en la familia, es una de las experiencias más significativas y trascendentales del ser humano, la noticia de que el niño o niña tiene una alteración congénita genera una ruptura emocional cargada de culpabilidad, sufrimiento y desorganización psicológica; primeramente, los padres usan sus recursos para establecer el vínculo con el bebe, posteriormente se ven necesitados de ir en la búsqueda de información y orientación para adaptarse y enfrentar dicha problemática.

Declaración de financiamiento y de conflicto de intereses:

El estudio fue financiado por la autora, quien declara no tener algún tipo de conflicto de interés en la investigación realizada.

\section{Correspondencia}

Gladys Norma Manturano Velásquez

Instituto Nacional Cardiovascular

Jr. Coronel Zegarra 417- Lima

Correo electrónico: norglama@hotmail.com 


\section{REFERENCIAS BIBLIOGRÁFICAS}

Alli, J. (2000). Plan de apoyo a la familia. Recuperado: http://www.unav.edu/matrimonioyfamilia/b/ Planfamilia/navarra/NavarraPlanII.pdf.

Barrera,F.(2011).Otros sistemas de apoyo social del enfermo crónico. Recuperado de: http://www.saludinnova.com/ communities/Primaria/blog/2011/07/12/otro.s.

Carmen, R. (2010). Apoyo emocional en la familia y en el trabajo psicoterapéutico. Recuperado de: http://www. angelfire.com/pe/actualidadpsi/apoyoemocional.html.

Colliere, M. E. (1993). Promover la vida de la práctica de las mujeres cuidadoras a los cuidados de enfermería. Editorial Interamericana, Madrid.

Domínguez, C. (1986). Los cuidados y la profesión de enfermería. Editorial Pirámide, Madrid.

Franco, R. (2004). Percepción del familiar del paciente crítico, respecto a la intervención de enfermería durante la crisis situacional en la Unidad de Cuidados Intensivos. Hospital Nacional Edgardo Rebagliati. M. (Tesis Licenciatura). Universidad Nacional Mayor de San Marcos. Lima- Perú.

Frydenberg, E. \& Lewis, R. (1996). ACS, Escala de afrontamiento para adolescentes. Madrid: TEA, adaptación española. Recuperado de: http://www.ejep. es/index.php/journal/article/view/65/pdf.

Gagnon, A. J. \& Wagnon, K., \& Covell, C. (1997). Apoyo emocional de enfermería en las mujeres. Brasil: Editorial labor.

Galindo, A. (2006). Proyecto de intervención psicológica en las familias de niños con cardiopatías congénitas: aplicación de un programa de relajación progresiva Madrid. Recuperado de: http://www.mapfre.com/ccm/ content/documentos/fundacion/salud/revista-medicina/ vol17-n1-art4-cardiopatias.pdf-

Hannah, M. \& Midlarski, E. (2005). Discapacidad intelectual. Recuperado de: http://hdl.handle.net/11181/3132?rd= 0031787327329623.

Kern, E., Moreno-Jiménez, B. (2007). Resiliencia de niños enfermos crónicos: aspectos teóricos. Psicología en Estudio, (12)1, 81-86.

Krausf, M. (1992). Efectos subjetivos de ayuda psicológica. Chile: Editorial Pontificia.

Langer, A., Campero, L. \& García (1998). Efectos psicosociales. México: Editorial British.

Lazarus, R. (2000). Estrés y emoción, manejo e implicaciones en nuestra salud. Editorial Bilbao.

Leiter, M.P., Gascón, S. y Martínez-Jarreta, B. (2008). A two process model of burnout: their relevance to spanish and canadian nurses. Psychology in Spain, 12(1), 37-45.

Llopis, J. (2011). Corazones de papel: patrones de cambio en las familias con niños con cardiopatías congénitas, España - Barcelona. Recuperado de: http://hdl.handle. net/10803/79124

Llopis, J. (2008). Redes sociales y salud. Una aplicación práctica, Editorial Divulga. Recuperado de: http://www. ankulegi.org/wp-content/uploads/2012/03/130111LlopisCan\%C3\%8C\%C6\%92ameras.pdf.

Matón, A. (1993). Cirugía cardíaca. Editorial Navarro, Madrid.

Mosquera, A. (2012). Estilos de afrontamiento en madres de hijos e hijas con alteraciones congénitas. Editorial ICSA-BD-UAEH, Colombia Huila.

Monroy, A. (2014) Cansancio del rol de cuidador: análisis de sus factores relacionados (II). Reduca (Enfermería, Fisioterapia y Podología, 6 (3): 261-292.

Pelaéz, C. (2014). Educación social en situaciones de riesgo y conflicto en Iberoamérica. Madrid: Universo.

Rodríguez, Mónica. (2007). Cardiopatía congénita. España: Editorial Iris.

Simponiato de Paula, E. (2008). Influencia del apoyo social para el fortalecimiento de las familias de niños con insuficiencia renal crónica de San Pablo. Recuperado de: http://www.scielo.br/pdf/rlae/v16n4/es_07.pdf.

Silva, J. (2005). Regulación emocional y psicopatología: el modelo de vulnerabilidad y resiliencia. Recuperado de: http://dx.doi.org/10.4067/S0717-92272005000300004.

Valdez, M. \& Flores, T. (1990). Psicología del estrés. Barcelona: Editorial Martínez.

Villagra, F. (2010). Cirugía torácica y cardiovascular. España: Editorial Luz.

Zaldívar, J. (2003). Amor y responsabilidad. Madrid: Editorial Razón.

Recibido: 24/05/2014 Aceptado: 04/10/2014 